\title{
Un rôle pour les UVA dans les dommages solaires causés à l'ADN
}

Les cancers de la peau, tant les mélanomes que les cancers épithéliaux, sont en progression constante depuis quelques années [1]. Les études épidémiologiques et biochimiques ont mis en cause le soleil et, plus particulièrement, les radiations ultraviolettes du spectre solaire dans l'apparition des cancers cutanés. Une exposition excessive et des coups de soleil reçus pendant l'enfance ont été respectivement impliqués dans la production des épithéliomas baso- ou spino-cellulaires, d'une part, et des mélanomes, d'autre part. Le spectre des radiations solaires terrestres se décompose, en fonction de leurs longueurs d'onde, en radiations ultraviolettes B (UVB, 280-320 nm) et A (UVA, $320-400 \mathrm{~nm}$ ), en lumière visible et en infrarouge. Les ultraviolets A et $\mathrm{B}$ ne représentent que $5,4 \%$ de l'énergie solaire; les UVB les plus lointains $(\lambda<295 \mathrm{~nm})$ et les UVC $(190-280 \mathrm{~nm})$ sont totalement absorbés par l'atmosphère terrestre.

On a longtemps admis que les radiations UVC, émises en laboratoire par des lampes dites «germicides" à vapeur de mercure, constituaient un modèle adapté à l'analyse de la cancérogenèse cutanée. Les nombreux travaux réalisés sur les UVC ont permis de déterminer la nature chimique des deux lésions majoritaires, à savoir les dimères de pyrimidine et les photoproduits (6-4), produites dans l'ADN, à la fois par les UVC et les UVB. Ces lésions de l'ADN, induites par les UVC ou par le rayonnement solaire, si elles ne sont pas éliminées par les divers systèmes de réparation de l'ADN, sont à l'origine de mutations [2]. Une véritable signature mutationnelle de l'irradia-

de la bactérie à l'homme: les UVC laissent, en effet, une empreinte spécifique dans le génome. Les mutations caractéristiques sont majoritairement des transitions de la paire G/C en A/T dans des sites bipyrimidiques, et des mutations de deux paires successives en tandem, CC $\rightarrow$ TT. Récemment, cette signature UV a été observée au niveau d'un gène suppresseur de tumeur dans des cancers cutanés non mélaniques [3, 4]. Le rôle des UVB solaires dans l'apparition de ces cancers cutanés est largement démontré. En revanche, celui des UVA est encore mal défini, alors qu'ils constituent plus de $90 \%$ de l'énergie ultraviolette solaire et pénètrent plus efficacement que les UVB dans les couches basales de l'épiderme où débute le processus de cancérisation. Afin de préciser le rôle respectif des UVA et des UVB dans le mécanisme du déclenchement de ce processus, nous avons entrepris des études comparatives de la spécificité des mutations induites par ces radiations à celles produites par les UVC et par la lumière solaire simulée [5-7].

Les mutations induites dans un gène endogène (le gène de l'adénine phosphoryl transférase ou APRT) de cellules de hamster, normales ou déficientes dans la voie de réparation de l'ADN par excision-resynthèse, ont été analysées. Lorsque la réparation est normale, un type de mutation représentatif des UVA, les transversions $\mathrm{A} / \mathrm{T} \rightarrow \mathrm{C} / \mathrm{G}$, a été mis en évidence. Cette classe de mutations est très peu fréquente dans les spectres de mutations induites par les UVC ou les UVB, mais représente $25 \%$ des mutations induites par la lumière solaire simulée [6]; les autres classes de muta- tions étaient similaires à celles induites par les UVB. On retrouve bien la signature des UVA dans les mutations engendrées par la lumière solaire simulée dans des cellules normales. En revanche, dans les cellules qui n'éliminent pas les lésions de l'ADN produites par les UV, les mutations induites par la lumière solaire peuvent, pour l'essentiel, être attribuées aux UVB [7]. L'absence de réparation a pour conséquence l'augmentation d'un type donné de mutations (les mutations doubles $\mathrm{CC} \rightarrow \mathrm{TT})$ et la disparition de la trace caractéristique des UVA. Les lésions induites par les UVA sont sans doutes «noyées » par celles, plus mutagènes et plus nombreuses car non réparées, induites par les UVB. En outre, les UVA sont aussi capables de donner lieu à des mutations $\mathrm{G} / \mathrm{C} \rightarrow \mathrm{A} / \mathrm{T}$, identiques à celles produites par les UVB. Notons que, dans ces cellules déficientes, les mutations produites par la lumière solaire sont du même type que celles observées dans les tumeurs de la peau de patients atteints de xeroderma pigmentosum, déficients en réparation de l'ADN [8]. Ces résultats ont été corroborés par une étude de mutagenèse entreprise sur un gène porté par un vecteur navette transfecté dans des cellules humaines [9]. Une cytotoxicité identique pour les UVA et les UVB a été observée pour un rapport de dose UVA/UVB égal à 700, [9]. La signification biologique de cette observation réside dans le fait que des doses 700 fois plus élevées d'UVA que d'UVB atteignent les cellules de la couche basale de peau après une exposition au soleil en présence d'écran UVB. De plus, dans ce système, à doses équitoxiques, les UVA sont au 
moins aussi mutagènes que les UVB. Il faut noter que les doses d'UVA et d'UVB utilisées dans ces différentes études [5-7, 9] correspondent à celles accumulées au niveau de la couche basale de la peau lors d'une exposition au soleil de quelques heures à 3 jours au solstice d'été.

Le rôle majeur des UVB solaires dans le déclenchement de la cancérogenèse cutanée est indiscutable. Au niveau des tumeurs cutanées non mélaniques, la signature UVA n'a pas encore été observée. Cependant, c'est seulement depuis une dizaine d'années, période inférieure au temps de latence nécessaire à l'apparition des cancers de la peau, qu'on constate le développement des salons de bronzage aux «UVA». De plus, jusqu'à ces toutes dernières années, les crèmes solaires étaient dépourvues de filtres à UVA. Aussi, sur la base de l'ensemble de ces résultats récents, on peut prédire que, dans les années à venir, la trace moléculaire de l'action des UVA se manifestera, selon toute probabilité, dans les nouvelles tumeurs décelées.

En conclusion, sans apporter une preuve absolue du rôle des UVA en cancérogenèse cutanée, les données expérimentales actuelles constituent une mise en garde contre l'usage abusif des UVA.

1. Dubertret L, Santus R, Morlière P. Ozone, sun, cancer : molecular and cellular mechanisms, prevention. Paris : Éditions Inserm, 1995.

2. Sarasin A. La réparation de l'ADN au centre de la biologie de la cellule. médecine/sciences 1994; $10: 951-2$.

3. Brash D, Rudolph J, Simon J, Lin A, McKenna G, Baden A, Ponten J. A role for sunlight in skin cancer : UV-induced p53 mutations in squamous cell carcinoma. Proc Natl Acad Sci USA 1991; 88: 10124-8.

4. Dumaz N, Stary A, Soussi T, Daya-Grosjean L, Sarasin A. Can we predict solar ultraviolet radiation as the causal event in human tumours by analysing the mutation spectra of the p53 gene? $\mathrm{Mu}$ tation Res 1994; 307: 375-86.

5. Drobetsky E, Moustacchi E, Glickman BW, Sage E. The mutational specificity of simulated sunlight at the aprt locus in rodent cells. Carcinogenesis $1994 ; 15: 1577-83$.

6. Drobetsky E, Turcotte J, Chateauneuf A. A role for ultraviolet A in solar mutagenesis. Proc Natl Acad Sci USA 1995; 92 : 2350-4.

E.S. 7. Sage E, Lamolet B, Brulay E, Moustacchi E,

E.M. Chateauneuf A, Drobetsky E. Mutagenic specificity of solar UV light in nucleotide excision repair-deficient rodent cells. Proc Natl Acad Sci USA 1996; 93 : 176-80.

8. Dumaz N, Drougard C, Sarasin A, Daya-Grosjean L. Specific UV-induced mutation spectrum in the p53 of skin tumors from DNA-repair-deficient xeroderma pigmentosum patients. Proc Natl Acad Sci USA 1993 ; 90 : 10529-33.

9. Robert C, Muel B, Benoit A, Dubertret L, Sara$\sin$ A, Stary A. Cell survival and shuttle vector mutagenesis induced by UVA and UVB radiation on a human cell line. I Invest Dermatol 1996 ; 106 : 721-8. 